\title{
Neurocognitive and behavioural outcomes in offspring exposed to maternal pre-existing diabetes: a systematic review and meta-analysis
}

\author{
Jennifer M. Yamamoto ${ }^{1,2,3}$ (D) Jamie L. Benham ${ }^{1,4} \cdot$ Deborah Dewey $^{4,5,6} \cdot$ J. Johanna Sanchez ${ }^{7} \cdot$ Helen R. Murphy ${ }^{8,9,10}$. \\ Denice S. Feig ${ }^{11,12,13} \cdot$ Lois E. Donovan ${ }^{1,2,3}$
}

Received: 15 February 2019 / Accepted: 10 May 2019 / Published online: 5 July 2019

(C) Springer-Verlag GmbH Germany, part of Springer Nature 2019

\begin{abstract}
Aims/hypothesis We performed a systematic review and meta-analysis to determine whether exposure to maternal pre-existing diabetes in pregnancy is associated with neurocognitive or behavioural outcomes in offspring.

Methods We searched MEDLINE, EMBASE, PsychINFO, the Cochrane Database of Systematic Reviews and Scopus for studies that examined any neurocognitive or behavioural outcomes in offspring of mothers with pre-existing diabetes in pregnancy in accordance with a published protocol (PROSPERO CRD42018109038). Title and abstract review, full-text review and data extraction were performed independently and in duplicate. Risk of bias was assessed using the Newcastle-Ottawa scale. Meta-analyses of summary measures were performed using random-effects models.

Results Nineteen articles including at least 18,681 exposed and 2,856,688 control participants were identified for inclusion. Exposure to maternal pre-existing diabetes in pregnancy was associated with a lower pooled intelligence quotient in the offspring (pooled weighted mean difference $-3.07[95 \% \mathrm{CI}-4.59,-1.55] ; I^{2}=0 \%$ ) and an increased risk of autism spectrum disorders (effect estimate 1.98 [95\% CI 1.46, 2.68]; $I^{2}=0 \%$ ). There was also an increased risk of attention deficit/hyperactivity disorder (pooled $\mathrm{HR}$ $1.36[95 \%$ CI $1.19,1.55] ; I^{2}=0 \%$ ), though this was based on only two studies. Although most studies were found to be high quality in terms of participant selection, in many studies, comparability of cohorts and adequacy of follow-up were sources of bias.

Conclusions/interpretation There is evidence to suggest that in utero exposure to maternal pre-existing diabetes is associated with some adverse neurocognitive and behavioural outcomes. It remains unclear what the role of perinatal factors is and the degree to which other environmental factors contribute to these findings.
\end{abstract}

Jennifer M. Yamamoto and Jamie L. Benham contributed equally to this study.

Electronic supplementary material The online version of this article (https://doi.org/10.1007/s00125-019-4923-0) contains peer-reviewed but unedited supplementary material, which is available to authorised users.

Jennifer M. Yamamoto

jennifer.yamamoto@ucalgary.ca

1 Department of Medicine, Cumming School of Medicine, University of Calgary, Richmond Road Diagnostic and Treatment Centre, 1820 Richmond Road SW, Calgary, AB T2T 5C7, Canada

2 Department of Obstetrics and Gynecology, Cumming School of Medicine, University of Calgary, Calgary, AB, Canada

3 Alberta Children's Hospital Research Institute, Cumming School of Medicine, University of Calgary, Calgary, AB, Canada

4 Department of Community Health Sciences, Cumming School of Medicine, University of Calgary, Calgary, AB, Canada

5 Department of Paediatrics, Cumming School of Medicine, University of Calgary, Calgary, AB, Canada
6 Owerko Centre at the Alberta Children's Hospital Research Institute, Cumming School of Medicine, University of Calgary, Calgary, AB, Canada

7 Sunnybrook Research Institute, Toronto, ON, Canada

8 Wolfson Diabetes and Endocrine Clinic, Cambridge University Hospitals NHS Foundation Trust, Cambridge, UK

9 Women's Health Academic Centre, Division of Women's and Children's Health, King's College London, London, UK

10 Norwich Medical School, University of East Anglia, Norwich, UK

11 Mount Sinai Hospital, Toronto, ON, Canada

12 Lunenfeld-Tanenbaum Research Institute, Toronto, ON, Canada

13 Department of Medicine, University of Toronto, Toronto, ON, Canada 


\section{Research in context}

\section{What is already known about this subject?}

- Pre-existing diabetes in pregnancy is associated with increased risk of poor obstetric/neonatal outcomes

- The adverse effect of in utero exposure to maternal pre-existing diabetes may extend beyond the neonatal period to include an increased risk of poor neurocognitive and behavioural outcomes, although the evidence is inconsistent

\section{What is the key question?}

- Is exposure to maternal pre-existing diabetes in utero associated with neurocognitive or behavioural problems in the offspring?

\section{What are the new findings?}

- Exposure to maternal pre-existing diabetes in pregnancy was associated with a lower pooled intelligence quotient and an increased risk of autism spectrum disorder

- While we found an increased risk of attention deficit/hyperactivity disorder in offspring of mothers with maternal pre-existing diabetes, this analysis was based on only two studies and should be interpreted with caution

- The relationship between maternal glycaemic control and offspring neurocognitive and behavioural outcomes is unclear

\section{How might this impact on clinical practice in the foreseeable future?}

- Heightened awareness of the association of exposure to pre-existing diabetes in utero with intelligence and autism spectrum disorder should contribute to a low threshold for investigating parental concerns in these children

Keywords ADHD · ASD · Autism · Behaviour · Childhood · Diabetes · IQ · Meta-analysis · Neurocognitive · Offspring · Pregnancy $\cdot$ Systematic review

$\begin{array}{ll}\text { Abbreviations } \\ \text { ADHD } & \text { Attention deficit/hyperactivity disorder } \\ \text { ASD } & \text { Autism spectrum disorder } \\ \text { GCSE } & \text { General Certificate of Secondary Education } \\ \text { IQ } & \text { Intelligence quotient } \\ \text { RR } & \text { Risk ratio }\end{array}$

\section{Introduction}

Diabetes in pregnancy is associated with an increased risk of obstetric and neonatal complications [1, 2]. Maternal preexisting diabetes is becoming increasingly prevalent in pregnancy. In type 1 diabetes, as many as one in two pregnancies will have a complication such as preterm delivery, offspring being large for gestational age, neonatal hypoglycaemia or neonatal intensive care unit admission [2, 3]. There is also an increased risk of less common but serious adverse outcomes such as congenital anomalies, stillbirth and neonatal death [4-6]. While the risk of short-term complications following diabetes in pregnancy is well established, the risk of longer-term adverse outcomes is not well defined.

It is hypothesised that in utero exposure to maternal diabetes, during this critical period of brain development, may lead to neurocognitive and behavioural difficulties persisting into child- and adulthood. Studies have evaluated exposure to maternal diabetes (all types) in relation to a wide range of neurocognitive and behavioural outcomes including intelligence quotient (IQ), school achievement, memory difficulties, attention deficit/hyperactivity disorder (ADHD) and autism spectrum disorder (ASD) [7-10]. However, the results are inconsistent, with some but not all studies showing an association between exposure to maternal diabetes (all types) and various neurocognitive and behavioural outcomes [7-10].

Systematic reviews have been performed to assess the association between maternal diabetes (all types) and childhood cognitive function and behaviour, but these reviews do not address the wide range of possible neurocognitive and behavioural outcomes evaluated in the literature and/or address the association between maternal diabetes and outcomes beyond childhood $[11,12]$. In addition, these reviews included gestational diabetes where the degree of hyperglycaemia is less severe and of shorter duration.

To address this knowledge gap, we performed a systematic review and meta-analysis to determine whether exposure to pre-existing diabetes (type 1, type 2 and other forms of preexisting diabetes such as monogenic diabetes) in pregnancy is 
associated with neurocognitive or behavioural outcomes in offspring at any age of assessment.

\section{Methods}

We performed a systematic review and meta-analysis in accordance with a published protocol (PROSPERO CRD42018109038). The Meta-analysis Of Observational Studies in Epidemiology (MOOSE) guidelines were used to report the findings [13].

Study question and eligibility This review aimed to address the following question: 'Is exposure to hyperglycaemia in utero associated with poor neurocognitive or behavioural outcomes in the offspring of mothers with pre-existing diabetes?'

Studies needed to address the following Population Exposure Comparison Outcome Design (PECOD) research question and meet all inclusion criteria to be eligible for inclusion in this review:

1) population: offspring of mothers with pre-existing diabetes in pregnancy;

2) exposure: maternal pre-existing diabetes in pregnancy (type 1 , type 2 and other forms such as monogenic diabetes);

3) comparison: no maternal diabetes (all types) or population-based standards;

4) outcomes: any neurocognitive or behavioural outcomes (e.g. IQ, Child Behaviour Checklist scores, Bayley Scale of Infant Development, Conners' Rating Scales for ADHD, etc.) beyond the neonatal period;

5) study design and methodology: we included only published randomised controlled trials or cohort studies that reported on original data. Only articles reported in English or French were included because these are the languages of the authors.

Search strategy and study selection The search was performed in duplicate using the search strategy outlined in the electronic supplementary material (ESM Methods). The search was performed on 12 September 2018. The search strategy was developed in collaboration with a medical librarian. The search was created using key words and Medical Subject Heading (MeSH) terms which were adjusted for each database. MEDLINE, EMBASE, PsychINFO, the Cochrane Database of Systematic Reviews and Scopus were searched using the identified search strategy for all available time periods. References of included studies and relevant reviews were hand-searched in duplicate to identify additional articles for inclusion. We conferred with experts in the field for the potential inclusion of additional articles.

Title and abstract review of all citations identified was performed to assess eligibility. Full-text review was completed for all citations identified at title and abstract review as eligible. Reasons for exclusion at full-text review were recorded. All stages of study selection were performed in duplicate by two independent reviewers. If multiple articles reported on the same cohort, they were only included if they reported different outcomes or the assessment was performed at different ages. If two articles reported data on the same outcome for the same cohort, the study that reported the measures most comprehensively was included. Agreement between reviewers was recorded and reported as a $\mathrm{K}$ statistic. Any disagreement between reviewers was resolved through consensus and/or in consultation with a third reviewer when necessary.

Data extraction Data were extracted independently and in duplicate using standardised forms. Extracted information included elements such as study design and other study characteristics, study population, number of participants, type of diabetes, neurocognitive and behavioural outcomes and their definitions, measures of association of maternal pre-existing diabetes with neurocognitive and behavioural outcomes, age at assessment, effect of maternal glycaemic control, adjustment for confounders, etc. Each reviewer independently assessed study quality. The Newcastle-Ottawa scale was used to assess the quality of nonrandomised studies [14]. Data management was performed using Covidence (Veritas Health Information Limited, Melbourne, Victoria, Australia, 2018) and Microsoft Excel (Microsoft Corporation, Redmond, WA, USA, version 15.30, 2017).

Data synthesis and analysis Meta-analyses were performed using random-effects models using the DerSimonian and Laird method. Summary measures of the effect size for standardised testing (i.e., IQ) and other neurocognitive and behavioural outcomes were expressed as mean differences, ORs, HRs or risk ratios (RRs) with $95 \%$ CIs where appropriate. ORs were substituted for RRs. All extracted ORs met the following criteria: (1) the incidence of the outcome was less than 10\%; and (2) the OR was between 0.5 and 2.5 [15]. Statistical heterogeneity was quantified using $I^{2}$ statistics. Pre-specified stratified analyses were performed by type of diabetes to explore reasons for heterogeneity; however, not all analyses pre-specified in our protocol could be performed given the lack of available data. Publication bias was assessed using graphical aids as appropriate. Statistical analyses were performed using STATA (StataCorp LP, College Station, TX, USA, version 14.1).

\section{Results}

A total of 4301 records were identified and their titles and abstracts reviewed (Fig. 1). Of these, 66 articles were chosen for full-text review. A total of 19 articles including more than 
Fig. 1 Preferred Reporting Items for Systematic Reviews and Meta-Analyses (PRISMA) flow diagram

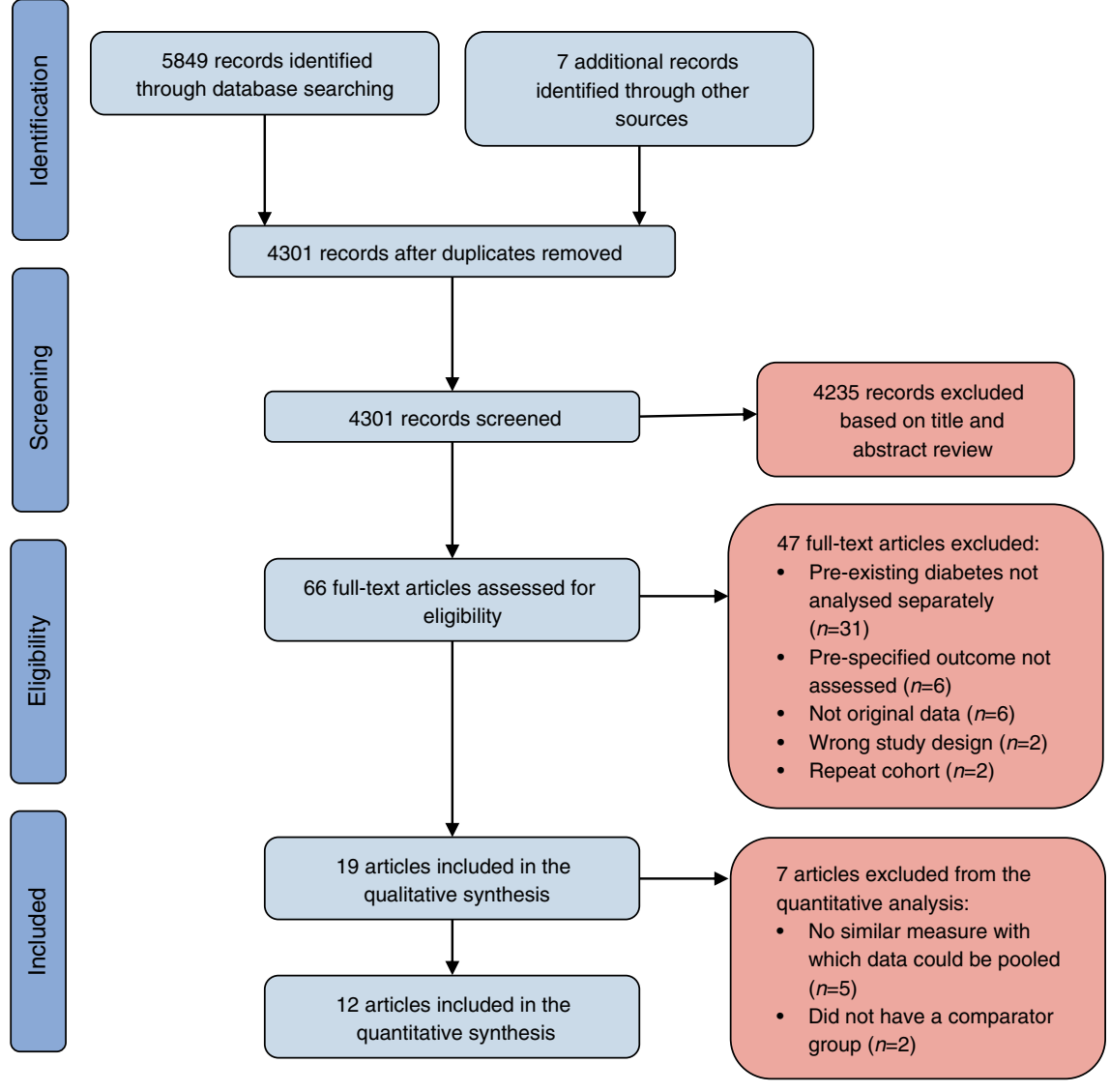

18,681 participants with pre-existing diabetes and 2,856,688 control participants were included in the qualitative analysis [7-10, 16-30] and 12 articles including more than 13,000 participants (two studies did not report an $\mathrm{n}$ for offspring with exposure to maternal pre-existing diabetes $[21,25])$ in the quantitative synthesis $[7,9,10,16,17,19-21,23,25-27$, $29,30]$. The literature search did not identify any randomised controlled trials with eligible follow-up data or data collected for secondary analysis that met inclusion criteria. Three of the included studies used the same cohort but reported on different outcomes; therefore, all were included in the review [17, $18,22]$. No studies knowingly included women with monogenic diabetes. The $\mathrm{k}$ statistic for inter-rater agreement was $0.70(95 \%$ CI $0.63,0.77)$ for the title and abstract review and $0.79(95 \%$ CI $0.62,0.95)$ for the full-text review.

Study characteristics The characteristics of included studies are summarised in Table 1 . Twelve studies were performed in Europe [7-9, 17, 18, 20-24, 27, 28] and seven in North America $[10,16,19,25,26,29,30]$. Sample sizes ranged from 40 to over $1,380,000$, with retrospective administrative database studies being largest. Age of assessment varied from 0.5 years to 27 years. Outcomes assessed included intelligence, behaviour, psychomotor development, developmental disorders such as ADHD and ASD, and psychiatric disorders.
Study quality assessment An assessment of study quality using the Newcastle-Ottawa scale for cohort studies is presented in Table 2 [14]. Most studies had high-quality participant selection $[7,9,10,16-19,21-23,25-30]$. Due to the nature of the outcome assessed, none of the studies had low risk of bias for 'demonstration that outcome not present at study onset'; therefore, this category was not included in the table. Studies were mixed in terms of comparability. In some studies, the exposed and unexposed cohorts were thought to be well matched and highly adjusted for potential confounders $[7,9,10,17,18,21-23,25,26]$, other studies had matching or adjusted analyses alone [20, 24, 27, 29] and some had no matching or adjustment at all $[8,16,19,28,30]$. Adequacy of follow-up was frequently unreported or thought to be a source of bias.

Exposure to maternal pre-existing diabetes and measures of intelligence We identified ten studies that examined the association of maternal pre-existing diabetes with measures of intelligence or intellectual disability [7-9, 17, 25-30]. Six were pooled in a meta-analysis for IQ [7, 9, 17, 27, 29, 30] (Table 3).

Two studies reported data that could not be pooled because they did not have a comparator group $[8,28]$. Persson et al assessed children of mothers with diabetes using the Terman- 


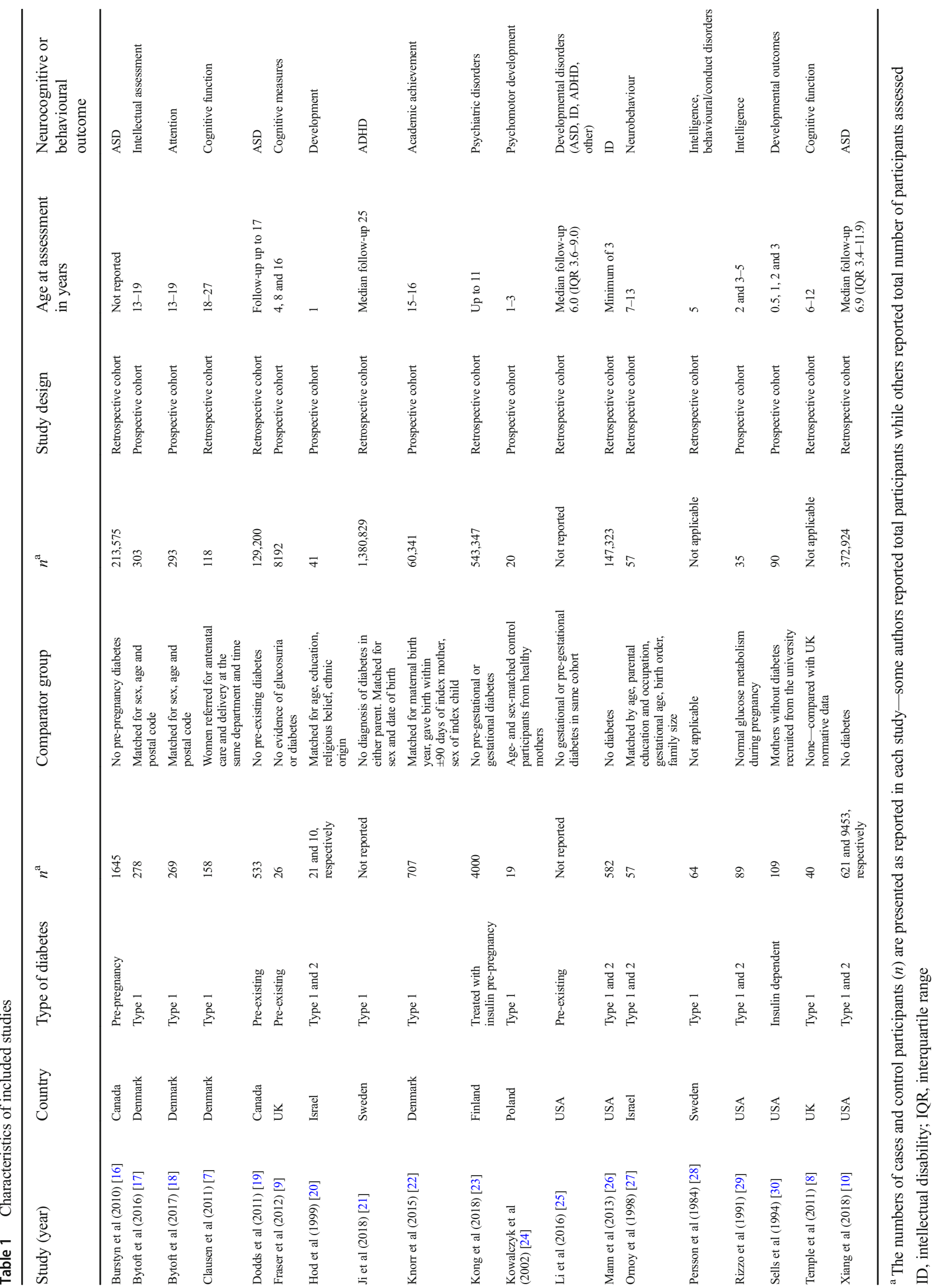


Table 2 Study quality assessment

\begin{tabular}{|c|c|c|c|c|c|c|c|}
\hline \multirow[t]{2}{*}{ Study (year) } & \multicolumn{3}{|l|}{ Selection } & \multirow{2}{*}{$\begin{array}{l}\text { Comparability of } \\
\text { cohorts based on } \\
\text { design or analysis }\end{array}$} & \multicolumn{3}{|l|}{ Outcome } \\
\hline & $\begin{array}{l}\text { Exposed cohort } \\
\text { represented }\end{array}$ & $\begin{array}{l}\text { Selection of } \\
\text { non-exposed } \\
\text { cohort }\end{array}$ & $\begin{array}{l}\text { Ascertainment } \\
\text { of exposure }\end{array}$ & & $\begin{array}{l}\text { Assessment } \\
\text { of outcome }\end{array}$ & $\begin{array}{l}\text { Follow-up long } \\
\text { enough for valid } \\
\text { use of assessment } \\
\text { tool }\end{array}$ & $\begin{array}{l}\text { Adequacy } \\
\text { of follow-up }\end{array}$ \\
\hline Burstyn et al (2010) [16] & $\star$ & $\star$ & * & - & $\star$ & $?$ & $\star$ \\
\hline Bytoft et al (2016) [17] & $\star$ & $\star$ & $\star$ & 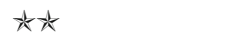 & $\star$ & $\star$ & - \\
\hline Bytoft et al (2017) [18] & $\star$ & $*$ & $\star$ & $* x$ & $\star$ & $\star$ & - \\
\hline Clausen et al (2011) [7] & $\star$ & $\star$ & $\star$ & $\star x$ & $\star$ & $\star$ & - \\
\hline Dodds et al (2011) [19] & $\star$ & $\star$ & $\star$ & - & $\star$ & $\star$ & $?$ \\
\hline Fraser et al (2012) [9] & $*$ & $\star$ & $*$ & $* *$ & $\star$ & $*$ & $?$ \\
\hline Hod et al (1999) [20] & $?$ & $?$ & $*$ & $\star$ & $?$ & * & $?$ \\
\hline Ji et al (2018) [21] & $*$ & $*$ & $\star$ & $* x$ & $\star$ & $*$ & $?$ \\
\hline Knorr et al (2015) [22] & $\star$ & $\star$ & $\star$ & 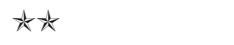 & $\star$ & $\star$ & $?$ \\
\hline Kong et al (2018) [23] & $\star$ & $\star$ & $\star$ & $* x$ & $\star$ & $\star$ & $\star$ \\
\hline Kowalczyk et al (2002) [24] & $?$ & $?$ & $\star$ & $\star$ & $?$ & $\star$ & $?$ \\
\hline Li et al (2016) [25] & $\star$ & $\star$ & $\star$ & $\star x$ & $\star$ & - & $?$ \\
\hline Mann et al (2013) [26] & $\star$ & $\star$ & $\star$ & $x+$ & $\star$ & - & - \\
\hline Ornoy et al (1998) [27] & $\star$ & $\star$ & $\star$ & $\star$ & $\star$ & $\star$ & $\star$ \\
\hline Persson et al (1984) [28] & $\star$ & Not applicable & $\star$ & Not applicable & $?$ & $\star$ & - \\
\hline Rizzo et al (1991) [29] & * & $\star$ & $\star$ & $\star$ & $*$ & $*$ & $?$ \\
\hline Sells et al (1994) [30] & $\star$ & $*$ & $*$ & - & * & $*$ & - \\
\hline Temple et al (2011) [8] & $?$ & Not applicable & $\star$ & - & $?$ & $\star$ & $?$ \\
\hline Xiang et al (2018) [10] & * & * & $\star$ & 站坛 & $\star *$ & $\star$ & $?$ \\
\hline
\end{tabular}

$\star$, low risk of bias; -, high risk of bias; ?, unclear risk of bias

${ }^{\text {a }}$ Two stars awarded if studies matched cases and control participants and reported adjusted estimates; one star awarded if they performed one or the other

Merril method to assess intelligence and reported that children of mothers with 'insulin-dependent diabetes' had an average IQ of 119 [28]. However, they did not provide a measure of error or have a comparison group. Temple et al assessed children of mothers with type 1 diabetes using the Wechsler Intelligence Scale for Children, version 4, and found that when compared with UK normative data there was no difference in overall IQ [8]. However, working memory scores were significantly lower in offspring exposed to maternal type 1 diabetes compared with UK normative data (Table 3).

A meta-analysis was performed for offspring IQ using data from six studies (Fig. 2). Because one study performed a stratified analysis by 'early entry' and 'late entry' cohorts (the exact definition of these cohorts was unclear), two main meta-analyses were performed: one with the early cohort and one with the late cohort [30]. In the main meta-analyses including the Sells 'early cohort', the pooled weighted mean difference was -3.07 (95\% CI $\left.-4.59,-1.55 ; I^{2}=0 \%\right)$, suggesting that offspring exposed to maternal pre-existing diabetes have a significantly lower IQ compared with control groups. The second main pooled analysis including the Sells 'late cohort' similarly found a lower IQ in offspring exposed to maternal pre-existing diabetes (mean difference -3.92 $\left.[95 \% \mathrm{CI}-5.55,-2.28] ; I^{2}=0 \%\right)$. A sensitivity analysis including only offspring of women with type 1 diabetes was performed and found a mean difference of $-4.62(95 \% \mathrm{CI}$ $-6.75,-2.50 ; I^{2}=0 \%$ ) compared with control participants. Both studies in women with type 1 diabetes only were in Denmark [7, 17]. It should be noted that Bytoft et al found a significantly lower IQ in offspring exposed to maternal type 1 diabetes after adjustment for confounders [17]. In contrast, Clausen et al reported that the significant difference between the groups was lost after adjustment [7]. The confounders and mediators adjusted for were similar between the two studies (ESM Table 1).

Exposure to maternal pre-existing diabetes and academic achievement Two studies assessed academic achievement; however, these data could not be pooled [9, 22]. Fraser et al studied a cohort from the UK and examined for differences in School Entry Assessment (age 4) and General Certificate of Secondary Education (GCSE) results (age 16) (Table 3) [9]. There was no significant difference in School Entry Assessment in offspring of mothers with pre-existing diabetes ( $n=21)$ compared with offspring of mothers without diabetes or glycosuria $(n=5804)$. However, offspring exposed to 


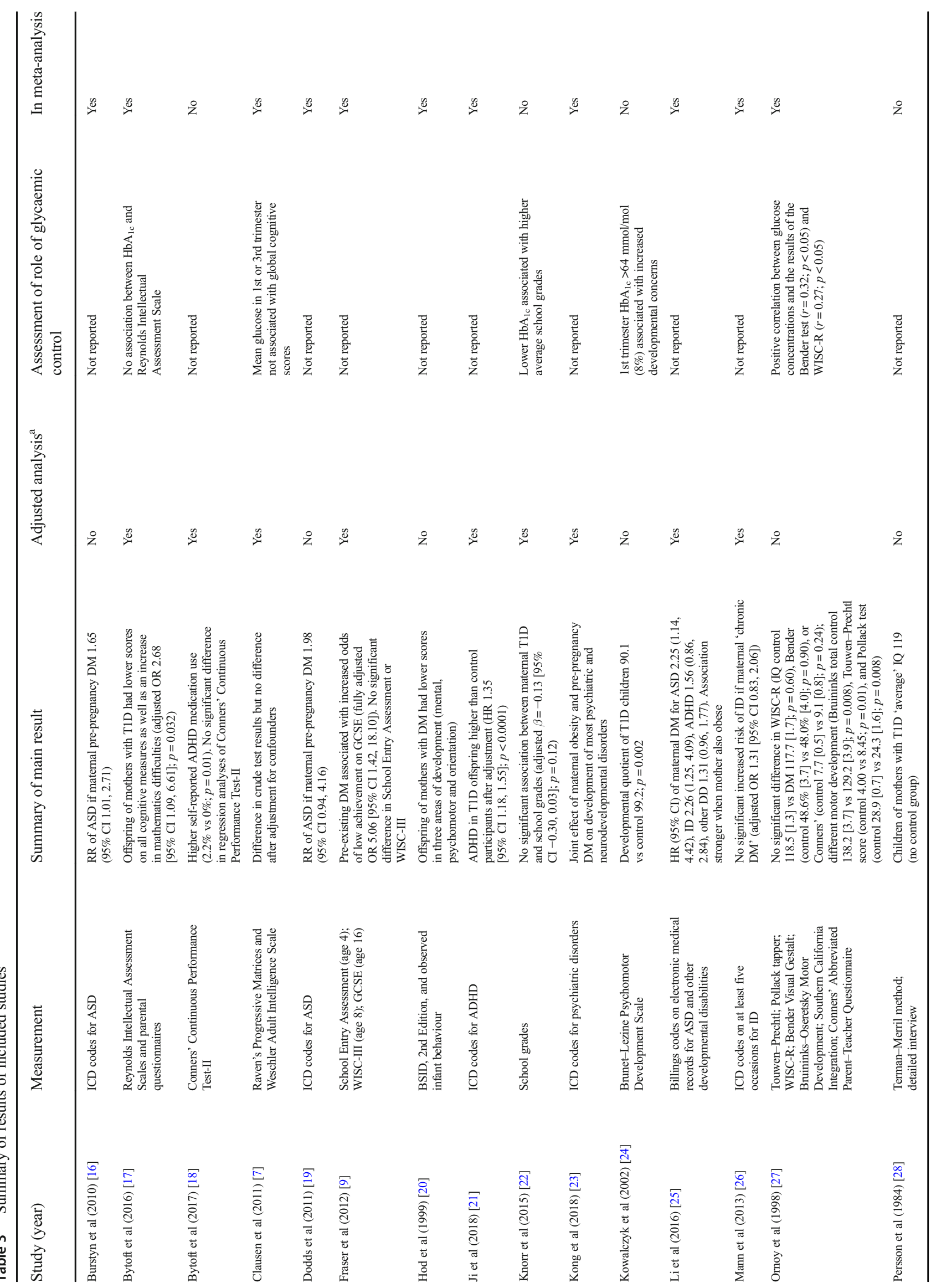



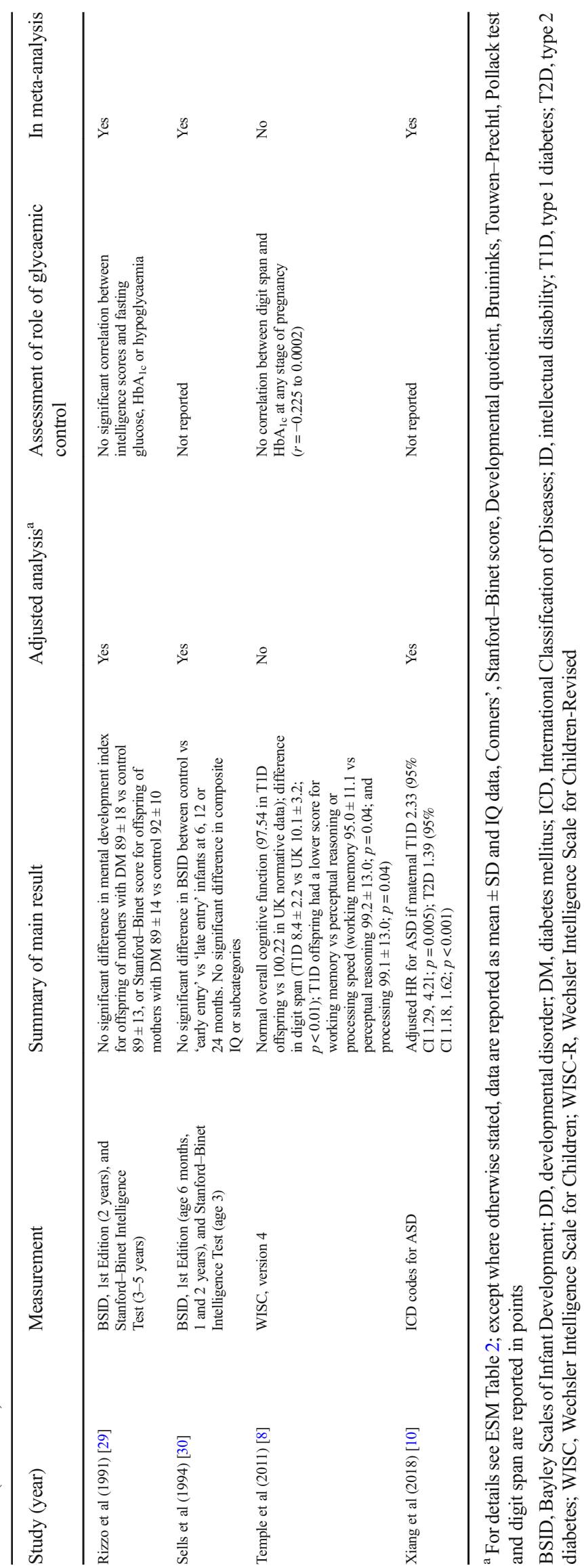

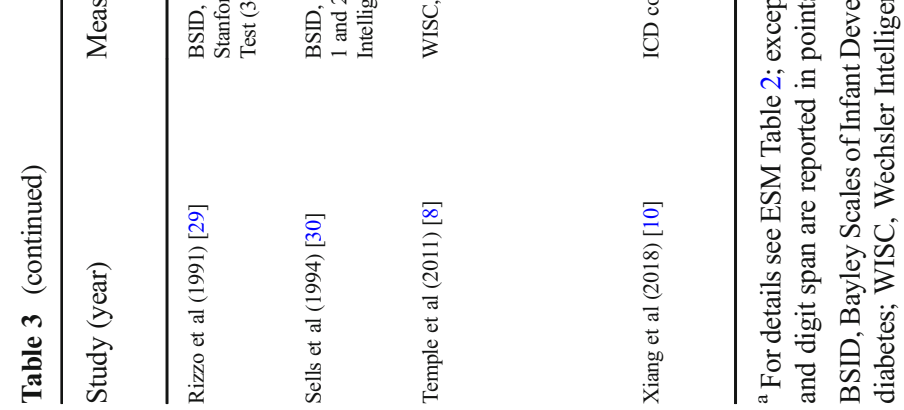
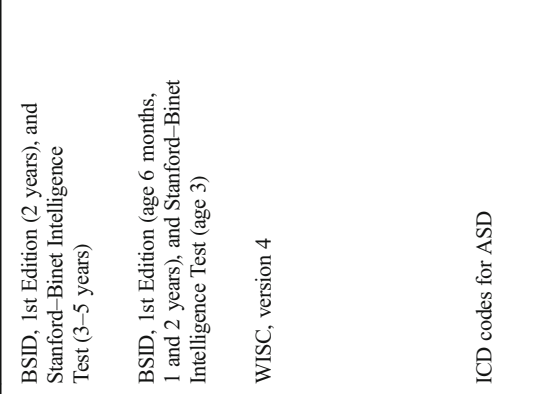
Fig. 2 Forest plots of IQ in offspring exposed to maternal pre-existing diabetes compared with control participants. (a)

Main analysis including the early cohort from Sells et al. (b) Main analysis including the late cohort from Sells et al. (c) Sensitivity analysis restricted to exposure to maternal type 1 diabetes. (a) and (b) include all types of preexisting diabetes; (c) is limited to studies including women with type 1 diabetes only. Weights are from random-effects analysis.

WMD, weighted mean difference of IQ points

a

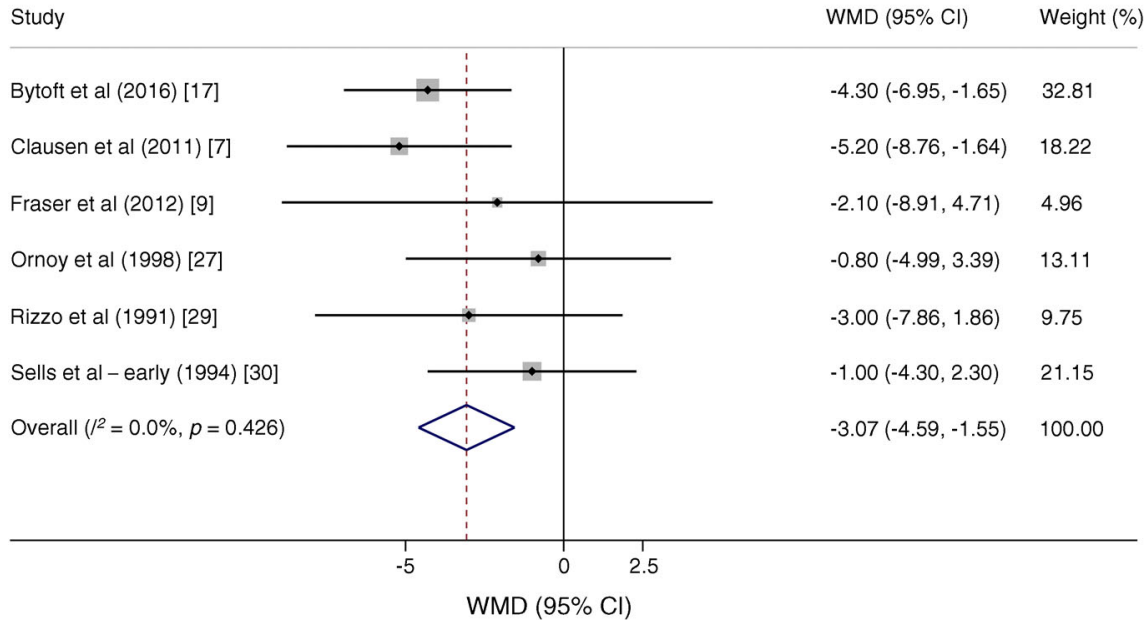

b

\begin{tabular}{|c|c|c|}
\hline Study & WMD $(95 \% \mathrm{Cl})$ & Weight $(\%$ \\
\hline Bytoft et al (2016) [17] & $-4.30(-6.95,-1.65)$ & 38.06 \\
\hline Clausen et al (2011) [7] & $-5.20(-8.76,-1.64)$ & 21.13 \\
\hline Fraser et al (2012) [9] & $-2.10(-8.91,4.71)$ & 5.76 \\
\hline Ornoy et al (1998) [27] & $-0.80(-4.99,3.39)$ & 15.20 \\
\hline Rizzo et al (1991) [29] & $-3.00(-7.86,1.86)$ & 11.31 \\
\hline Sells et al - late (1994) [30] - & $-7.00(-12.59,-1.41)$ & 8.55 \\
\hline Overall $\left(/^{2}=0.0 \%, p=0.510\right)$ & $-3.92(-5.55,-2.28)$ & 100.00 \\
\hline & & \\
\hline
\end{tabular}

C Study WMD $(95 \% \mathrm{Cl}) \quad$ Weight $(\%)$ Bytoft et al (2016) [17] Clausen et al (2011) [7] Overall $\left(/^{2}=0.0 \%, p=0.691\right)$

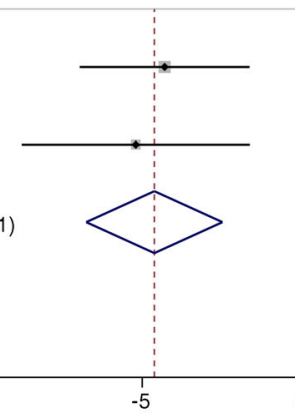

WMD $(95 \% \mathrm{Cl})$

diabetes had greater odds of having low achievement on their GCSEs (adjusted OR 5.06 [95\% CI 1.42, 18.10]). Conversely, Knorr et al did not find a significant difference in academic achievement in primary school performance in Denmark between offspring of mothers with type 1 diabetes and matched control participants [22].
Exposure to maternal pre-existing diabetes and child development Four studies looked at developmental measures in childhood [20,24, 29, 30], three of which could be pooled in a meta-analysis $[20,29,30]$ (Table 3). Kowalczyk et al used the Brunet-Lezine Psychomotor Development Scale, which could not be pooled with the Bayley Scales of Infant 
Development (1st and 2nd Editions) used in the other three studies [24]. Kowalczyk et al found that children of mothers with type 1 diabetes had lower scores, with a developmental quotient of 90.1 in offspring of mothers with type 1 diabetes vs 99.2 in the control group; $p=0.002$.

The meta-analysis of studies using the Bayley Scales of Infant Development, 1st and 2nd Editions, is found in ESM Figs 1, 2. When multiple assessment times were available, we chose the oldest age group as it is a better predictor of later IQ [31]. There was no significant difference in Mental Developmental Index (pooled mean difference -2.79 [95\% CI $-7.82,2.24]$ using the 'early entry' Sells et al cohort, and -4.50 [95\% CI $-9.06,0.06]$ using the 'late entry' cohort) (ESM Fig. 1a, b) [30]. There was also no significant difference in Psychomotor Developmental Index (pooled mean difference $-5.95[95 \%$ CI $-14.16,2.26]$ using the 'early entry' Sells et al cohort and $-4.62[95 \% \mathrm{CI}-16.73,7.50]$ using the 'late entry' cohort) (ESM Fig. 2a, b) [30].

Exposure to maternal pre-existing diabetes and ASD Five studies examining the association between maternal preexisting diabetes and ASD were found. All were large database studies [10, 16, 19, 23, 25] (Table 3). Exposure to maternal pre-existing diabetes was associated with an increased risk of ASD. When women with type 1 diabetes from the Xiang et al cohort were included, the effect estimate was $1.98(95 \%$ CI 1.46, 2.68; $I^{2}=0 \%$ ) (Fig. 3) [10]. When the analysis was re-run, substituting women with type 2 diabetes from the same Xiang et al cohort for Xiang's cohort of women with type 1 diabetes, this effect estimate was 1.46 (95\% CI 1.26, 1.69; $I^{2}=0 \%$ ) (Fig. 3). Because Kong et al performed a stratified analysis by BMI, it could not be included in the overall analysis [23].
Exposure to maternal pre-existing diabetes and ADHD Four studies assessed the relationship between exposure to maternal pre-existing diabetes and ADHD [18, 21, 23, 25] (Table 3), three of which were included in a meta-analysis [21, 23, 25]. The Bytoft et al study was not included as it assessed for ADHD by history of medication use and used the Conners' Continuous Performance test version 2 but did not report an RR, OR or HR [18]. They found that, while there was higher self-reported ADHD medication use (2.2 vs $0 \% ; p=0.01)$, there was no significant difference in regression analyses with the Conners' Continuous Performance test.

Pooled adjusted HR for the diagnosis of ADHD after exposure to maternal pre-existing diabetes was $1.36(95 \% \mathrm{CI}$ $1.19,1.55 ; I^{2}=0 \%$ ) (Fig. 4). Again, the data from Kong et al were not included as they were only presented as a stratified analysis [23].

Assessment of the role of maternal glycaemic control Seven studies reported on the association of maternal glycaemic control with neurocognitive or behavioural measures in offspring [7, 8, 17, 22, 24, 27, 29] (Table 3). Only three studies found glycaemic control to be associated with offspring outcomes $[22,24,27]$. Knorr et al noted that lower $\mathrm{HbA}_{1 \mathrm{c}}$ was associated with higher average school grades; however, they highlight the strong correlation between maternal education level and glycaemic control [22]. Kowalczyk et al found that a first trimester $\mathrm{HbA}_{1 \mathrm{c}}$ of $>64 \mathrm{mmol} / \mathrm{mol}(8 \%)$ was associated with an increased risk of developmental abnormalities [24]. Lastly, Ornoy et al noted a positive correlation between maternal glucose concentrations and results on the Bender Visual Gestalt test (a test used to evaluate hand-eye coordination) and Wechsler Intelligence Scales for Children, Revised [27]. Despite adjustment for parental education, Sells et al
Fig. 3 Forest plot of ASD in offspring exposed to maternal pre-existing diabetes compared with control participants. This analysis includes all types of preexisting diabetes, and includes a pooling of RR and HR. Weights are from random-effects analysis. ES, effect size

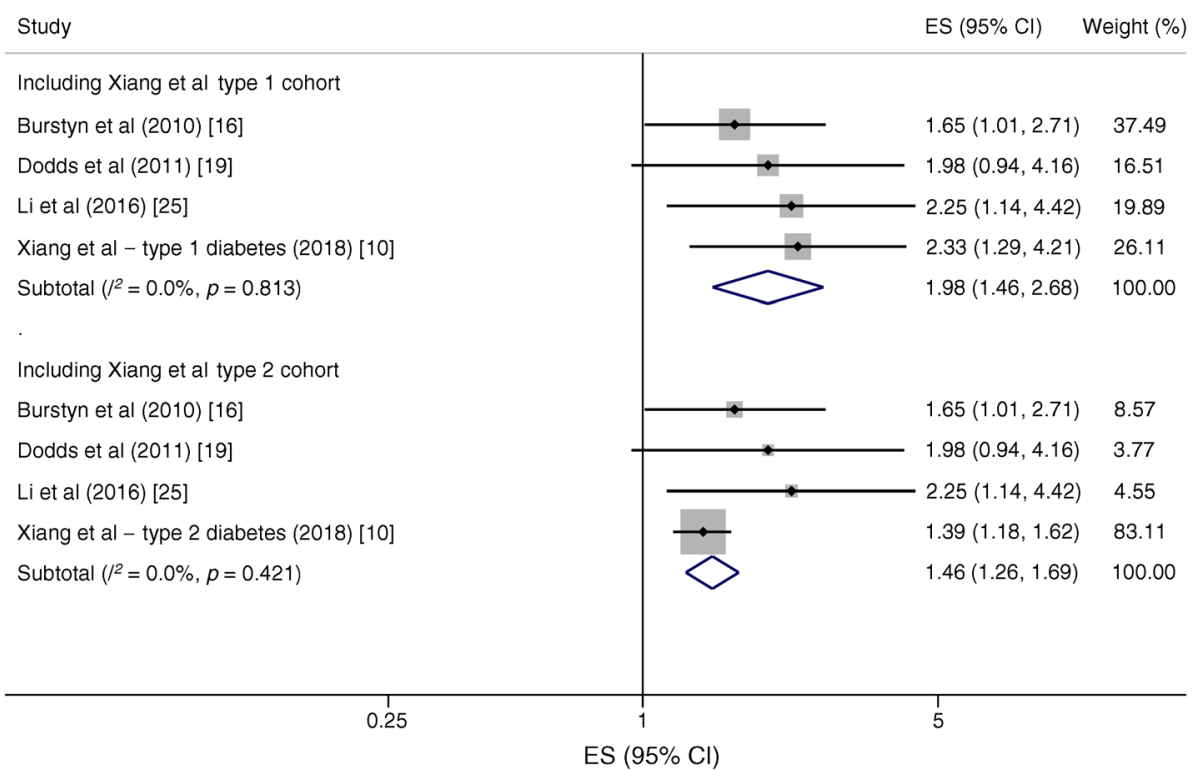


Fig. 4 Forest plot of attention deficit disorders in offspring exposed to maternal pre-existing diabetes compared with control participants. This analysis includes all types of pre-existing diabetes, and includes a pooling of HR for attention deficit disorders. Weights are from random-effects analysis. ES, effect size

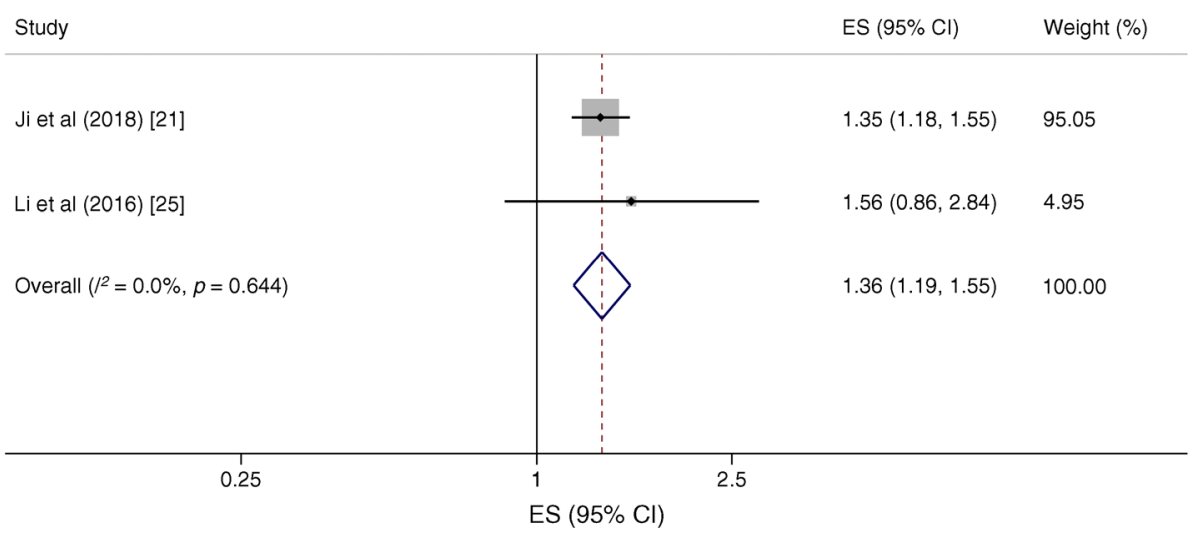

identified poorer $(p<0.05)$ scores in the Peabody Picture Vocabulary Test and the Vineland Communication Subscale but no difference in the Mean Length of Utterance at 3 years among children of mothers with 'late study entry' who had significantly higher first and second trimester $\mathrm{HbA}_{1 \mathrm{c}}$ compared with mothers with 'early study entry' [30].

Evaluation for small-study effect and publication bias An assessment for small-study effect was only performed on the metaanalysis of IQ since all other pooled analyses had very few studies. The funnel plots for the main analysis (Fig. 2a, b) were symmetrical, suggesting that there was no evidence of smallstudy effects (ESM Fig. 3a, b). However, given that the effect estimates are similar across studies we cannot rule out the possibility that larger studies are more often published, whereas smaller studies are only published if they report positive findings.

For the ASD meta-analysis, most studies included had a lower CI limit that was close to the null. This may indicate a bias to publish studies with a significant finding.

\section{Discussion}

This systematic review and meta-analysis found that in utero exposure to maternal pre-existing diabetes was associated with poorer neurocognitive and behavioural outcomes including lower pooled IQ, an increased risk of ASD and an increased risk of ADHD. It was unclear whether exposure was associated with lower academic achievement or lower infant development scores.

There was a significantly lower pooled IQ in offspring exposed to maternal pre-existing diabetes compared with comparator groups. While the overall effect $(-3.07$ to -3.92 IQ points) in an individual child may not be clinically meaningful, when applied at a population level this difference is noteworthy and demands further investigation. It is important to note that this pooled IQ is unadjusted. There are many important potential confounders in examining IQ such as parental IQ/education, socioeconomic status, gestational age at delivery and perinatal complications such as preterm delivery and neonatal hypoglycaemia [32] which may be different in offspring of mothers with diabetes compared with those without.

Some studies matched for some important confounders, though residual confounding likely exists even after matching $[7,17,27]$. It is noteworthy that the two studies that were limited to women with type 1 diabetes had the largest difference in IQ between groups [7, 17]. Interestingly, in the study by Bytoft et al [17] this difference remained significant after adjustment for many confounders, whereas in the study by Clausen et al, this difference was lost [7]. This may be due to the larger sample size in the Bytoft cohort $(n=578)$ vs the Clausen cohort $(n=276)$, as both were in Denmark and had very similar adjusted analyses performed.

Offspring exposure to maternal pre-existing diabetes was associated with an increased risk of ASD and ADHD in most analyses in this review. This is consistent with previously published meta-analyses examining ASD [33, 34]. The impact of the ASD effect size is currently unknown but is large enough to prompt further investigation of this association. To our knowledge, no other published meta-analyses have examined the risk of ADHD in this population. The risk of ASD with maternal obesity has been previously reported [35]. However, the associations between these outcomes and maternal pre-existing diabetes and how they are modulated by maternal blood glucose levels are interesting and merit further study. We identified one study that examined the risk of attention deficits in a cohort including only type 1 diabetes offspring [18]. It found a significant difference in ADHD medication use but no difference in direct measures. However, these results may be a result of the much smaller sample size ( $n=269$ offspring) in this prospective cohort study compared with larger retrospective database studies.

Our review was unable to identify evidence definitively suggesting a difference in academic achievement or Bayley Scale of Infant Development scores between infants exposed to maternal pre-existing diabetes and those who were not. Only two studies investigated difference in academic achievement and the results were mixed. The study by Fraser et al was large but only included 26 offspring exposed to maternal preexisting diabetes [9]. The study by Knorr et al had a larger 
number of diabetes-exposed offspring $(n=707)$ and did not find a difference between groups [22]. Only three studies, all performed over 20 years ago, examined performance on the Bayley Scale of Infant Development, 1st and 2nd Editions $[20,29,30]$. While these studies found lower scores on both the mental and psychomotor development indexes, none were statistically significant. The small number of studies as well as the small number of participants included $(n=72-199)$ may have contributed to this negative result.

The literature was also discordant regarding the role of maternal glycaemic control and offspring neurocognitive and behavioural outcomes. Only seven of the 19 studies included in this review reported on this and the results were variable $[7,8,17,22$, 24, 27, 29]. Assessment of glycaemic control in these studies was limited to $\mathrm{HbA}_{1 \mathrm{c}}$ or mean glucose, both of which are surrogate measures of glycaemic control and are inadequate in assessing daily glucose excursions, especially during pregnancy. There may also be important confounding in relating glycaemic control measures to intelligence and/or academic achievement. Knorr et al, for example, found that lower $\mathrm{HbA}_{1 \mathrm{c}}$ was strongly correlated with maternal education [22]. For this reason, it is especially important to adjust for maternal education and socioeconomic status in future studies. A more detailed examination of the relationship between maternal glycaemic control and offspring neurocognitive and behavioural outcomes using continuous glucose monitoring data should be performed. Continuous glucose monitoring allows for an assessment of direct fetal exposure to maternal hyper- and hypoglycaemia and may be more informative for assessing in utero glycaemic exposure.

Our review has both similarities and differences to previously published reviews in the area $[11,12]$. Unlike recent systematic reviews, ours is restricted to offspring exposed to preexisting diabetes and does not include women with gestational diabetes, which is a less severe form of glycaemic disturbance and of shorter duration. Our review also included studies that assessed outcomes into adulthood and seven additional studies published in the last few years. As with our systematic review and meta-analysis, both of these reviews highlight the important role of confounding when interpreting the data [11, 12].

This systematic review and meta-analysis benefits from its rigorous methodology and broad inclusion criteria, allowing for a comprehensive assessment of existing literature. To our knowledge, it is also the first review to perform a pooled analysis of the risk for ADHD in offspring exposed to maternal preexisting diabetes. It is further strengthened by our inclusion of only women with pre-existing diabetes in pregnancy. However, there are also limitations to acknowledge. Diabetes was included in our search terms and therefore we may have missed studies that were not specific to diabetes $[16,19]$. We have mitigated that limitation with a reference review of all included studies and relevant reviews. Many of the prospective cohort studies had inadequate or unreported assessments of neurocognitive and behavioural outcomes, which makes them particularly prone to bias. Also, they may be underpowered to show a difference in outcomes. Unfortunately, most studies did not report an assessment of power. In addition, many studies were unadjusted or minimally adjusted. Knowing that there are many important confounders, these findings must be interpreted with caution. Additionally, the pooled analysis for ADHD included only two studies of vastly varying sample sizes and, therefore, this analysis must be interpreted carefully. Heterogeneity between studies calculated using the $I^{2}$ statistic should also be interpreted cautiously as our power was limited given the small number of studies included in each presented analysis. Lastly, few studies reported on the role of glycaemic control and no studies reported detailed measures of glycaemic control.

When taken together, these data suggest there is a signal that exposure to maternal pre-existing diabetes is associated with adverse neurocognitive and behavioural outcomes past the neonatal period. However, caution must be exercised when interpreting these findings. While some of these analyses were highly adjusted, it remains unclear what the roles of perinatal factors such as preterm delivery and neonatal hypoglycaemia are and the degree to which other environmental factors may contribute to these findings. It is essential that future studies focus on identifying potentially modifiable risk factors such as maternal glycaemic control, which may assist women and their care providers in preventing neurocognitive or behavioural complications associated with prenatal exposure to maternal pre-existing diabetes.

Acknowledgements The authors would like to thank H. L. Robertson (University of Calgary, Calgary, Canada) for her expert advice in developing our search strategy. We also thank L. Lau (University of Calgary, Calgary, Canada) for her contribution to data extraction.

Data availability All data used in this systematic review and metaanalysis are published and referenced.

Funding This research received no specific grant from any funding agency in the public, commercial or not-for-profit sectors.

Duality of interest The authors declare that there is no duality of interest associated with this manuscript.

Contribution statement JMY, JLB, LED, DSF, HRM, JJS and DD conceived and designed the study. JMY and JLB performed the search, data extraction and data analysis. JMY wrote the initial manuscript draft. JMY, JLB, LED, DSF, HRM, JJS and DD all participated in the critical revision of the manuscript for important intellectual content and approved the final version. JMY is the guarantor of this work, had full access to all of the study data and takes responsibility for the integrity of the data.

\section{References}

1. Tennant PW, Glinianaia SV, Bilous RW, Rankin J, Bell R (2014) Pre-existing diabetes, maternal glycated haemoglobin, and the risks of fetal and infant death: a population-based study. Diabetologia 57(2):285-294. https://doi.org/10.1007/s00125-013-3108-5 
2. Murphy HR, Bell R, Cartwright $C$ et al (2017) Improved pregnancy outcomes in women with type 1 and type 2 diabetes but substantial clinic-to-clinic variations: a prospective nationwide study. Diabetologia 60(9):1668-1677. https://doi.org/10.1007/s00125017-4314-3

3. Feig DS, Donovan LE, Corcoy R et al (2017) Continuous glucose monitoring in pregnant women with type 1 diabetes (CONCEPTT): a multicentre international randomised controlled trial. Lancet 390(10110):2347-2359. https://doi.org/10.1016/S0140-6736(17) 32400-5

4. Evers IM, de Valk HW, Visser GH (2004) Risk of complications of pregnancy in women with type 1 diabetes: nationwide prospective study in the Netherlands. BMJ 328(7445):915. https://doi.org/10. 1136/bmj.38043.583160.EE

5. Macintosh MC, Fleming KM, Bailey JA et al (2006) Perinatal mortality and congenital anomalies in babies of women with type 1 or type 2 diabetes in England, Wales, and Northern Ireland: population based study. BMJ 333(7560):177. https://doi.org/10.1136/ bmj.38856.692986.AE

6. Mackin ST, Nelson SM, Kerssens JJ et al (2018) Diabetes and pregnancy: national trends over a 15 year period. Diabetologia 61(5):1081-1088. https://doi.org/10.1007/s00125-017-4529-3

7. Clausen TD, Mortensen EL, Schmidt L et al (2011) Cognitive function in adult offspring of women with type 1 diabetes. Diabet Med 28(7):838-844. https://doi.org/10.1111/j.1464-5491.2011.03300.x

8. Temple RC, Hardiman M, Pellegrini M, Horrocks L, MartinezCengotitabengoa MT (2011) Cognitive function in 6- to 12-yearold offspring of women with type 1 diabetes. Diabet Med 28(7): 845-848. https://doi.org/10.1111/j.1464-5491.2011.03285.x

9. Fraser A, Nelson SM, Macdonald-Wallis C, Lawlor DA (2012) Associations of existing diabetes, gestational diabetes, and glycosuria with offspring IQ and educational attainment: the Avon Longitudinal Study of Parents and Children. Exp Diabetes Res 2012:963735

10. Xiang AH, Wang X, Martinez MP, Page K, Buchanan TA, Feldman RK (2018) Maternal type 1 diabetes and risk of autism in offspring. JAMA 320(1):89-91. https://doi.org/10.1001/jama.2018.7614

11. Adane AA, Mishra GD, Tooth LR (2016) Diabetes in pregnancy and childhood cognitive development: a systematic review. Pediatrics 137(5):e20154234. https://doi.org/10.1542/peds.20154234

12. Camprubi Robles M, Campoy C, Garcia Fernandez L, LopezPedrosa JM, Rueda R, Martin MJ (2015) Maternal diabetes and cognitive performance in the offspring: a systematic review and meta-analysis. PLoS One 10(11):e0142583. https://doi.org/10. 1371/journal.pone. 0142583

13. Stroup DF, Berlin JA, Morton SC et al (2000) Meta-analysis of observational studies in epidemiology: a proposal for reporting. Meta-analysis Of Observational Studies in Epidemiology (MOOSE) group. JAMA 283(15):2008-2012. https://doi.org/10. 1001/jama.283.15.2008

14. Wells G, Shae B, O'Connell D et al. (2013) The Newcastle-Ottawa Scale (NOS) for assessing the quality of nonrandomised studies in meta-analyses. Available from http://www.ohri.ca/programs/ clinical_epidemiology/oxford.asp. Accessed 22 Aug 2018

15. Zhang J, Yu KF (1998) What's the relative risk? A method of correcting the odds ratio in cohort studies of common outcomes. JAMA 280(19):1690-1691. https://doi.org/10.1001/jama.280.19. 1690

16. Burstyn I, Sithole F, Zwaigenbaum L (2010) Autism spectrum disorders, maternal characteristics and obstetric complications among singletons born in Alberta, Canada. Chronic Dis Can 30(4):125134

17. Bytoft B, Knorr S, Vlachova Z et al (2016) Long-term cognitive implications of intrauterine hyperglycemia in adolescent offspring of women with type 1 diabetes (the EPICOM study). Diabetes Care 39(8):1356-1363. https://doi.org/10.2337/dc16-0168

18. Bytoft B, Knorr S, Vlachova Z et al (2017) Assessment of attention deficits in adolescent offspring exposed to maternal type 1 diabetes. PLoS One 12(1):e0169308. https://doi.org/10.1371/journal.pone. 0169308

19. Dodds L, Fell DB, Shea S, Armson BA, Allen AC, Bryson S (2011) The role of prenatal, obstetric and neonatal factors in the development of autism. J Autism Dev Disord 41(7):891-902. https://doi. org/10.1007/s10803-010-1114-8

20. Hod M, Levy-Shiff R, Lerman M, Schindel B, Ben-Rafael Z, Bar J (1999) Developmental outcome of offspring of pregestational diabetic mothers. J Pediatr Endocrinol Metab 12(6):867-872

21. Ji J, Chen T, Sundquist J, Sundquist K (2018) Type 1 diabetes in parents and risk of attention deficit/hyperactivity disorder in offspring: a population-based study in Sweden. Diabetes Care 41(4): 770-774. https://doi.org/10.2337/dc17-0592

22. Knorr S, Clausen TD, Vlachova $Z$ et al (2015) Academic achievement in primary school in offspring born to mothers with type 1 diabetes (the EPICOM study): a register-based prospective cohort study. Diabetes Care 38(7):1238-1244. https://doi.org/10.2337/ dc15-0223

23. Kong L, Norstedt G, Schalling M, Gissler M, Lavebratt C (2018) The risk of offspring psychiatric disorders in the setting of maternal obesity and diabetes. Pediatrics 142(3):e20180776. https://doi.org/ 10.1542/peds.2018-0776

24. Kowalczyk M, Ircha G, Zawodniak-Szalapska M, Cypryk K, Wilczynski J (2002) Psychomotor development in the children of mothers with type 1 diabetes mellitus or gestational diabetes mellitus. J Pediatr Endocrinol Metab 15(3):277-281

25. Li M, Fallin MD, Riley A et al (2016) The association of maternal obesity and diabetes with autism and other developmental disabilities. Pediatrics 137(2):e20152206. https://doi.org/10.1542/peds. 2015-2206

26. Mann JR, Pan C, Rao GA, McDermott S, Hardin JW (2013) Children born to diabetic mothers may be more likely to have intellectual disability. Matern Child Health J 17(5):928-932. https://doi.org/10.1007/s10995-012-1072-1

27. Ornoy A, Ratzon N, Greenbaum C, Peretz E, Soriano D, Dulitzky M (1998) Neurobehaviour of school age children born to diabetic mothers. Arch Dis Child Fetal Neonatal Ed 79(2):F94-F99. https:// doi.org/10.1136/fn.79.2.F94

28. Persson B, Gentz J (1984) Follow-up of children of insulindependent and gestational diabetic mothers. Neuropsychological outcome. Acta Paediatr Scand 73(3):349-358. https://doi.org/10. 1111/j.1651-2227.1994.tb17747.x

29. Rizzo T, Metzger BE, Burns WJ, Burns K (1991) Correlations between antepartum maternal metabolism and intelligence of offspring. N Engl J Med 325(13):911-916. https://doi.org/10.1056/ NEJM199109263251303

30. Sells CJ, Robinson NM, Brown Z, Knopp RH (1994) Long-term developmental follow-up of infants of diabetic mothers. J Pediatr 125(1):S9-S17. https://doi.org/10.1016/S0022-3476(94)70170-9

31. Potharst ES, Houtzager BA, van Sonderen L et al (2012) Prediction of cognitive abilities at the age of 5 years using developmental follow-up assessments at the age of 2 and 3 years in very preterm children. Dev Med Child Neurol 54(3):240-246. https://doi.org/10. $1111 / j .1469-8749.2011 .04181 . x$

32. Shah R, Harding J, Brown J, McKinlay C (2018) Neonatal glycaemia and neurodevelopmental outcomes: a systematic review and meta-analysis. Neonatology 115:116-126

33. Xu G, Jing J, Bowers K, Liu B, Bao W (2014) Maternal diabetes and the risk of autism spectrum disorders in the offspring: a systematic review and meta-analysis. J Autism Dev Disord 44(4):766775. https://doi.org/10.1007/s10803-013-1928-2 
34. Wan H, Zhang C, Li H, Luan S, Liu C (2018) Association of maternal diabetes with autism spectrum disorders in offspring: a systemic review and meta-analysis. Medicine 97(2):e9438. https:// doi.org/10.1097/MD.0000000000009438

35. Li YM, Ou JJ, Liu L, Zhang D, Zhao JP, Tang SY (2016) Association between maternal obesity and autism spectrum disorder in offspring: a meta-analysis. J Autism Dev Disord 46(1):95-102. https://doi.org/10.1007/s10803-015-2549-8

Publisher's note Springer Nature remains neutral with regard to jurisdictional claims in published maps and institutional affiliations. 